Original Article

\title{
Manual Lymph Drainage Attenuates Frontal EEG Asymmetry in Subjects with Psychological Stress: A Preliminary Study
}

\author{
Jung-Myo Shim, PhD ${ }^{1)}$, Sung-Joong KIm, PT, $\mathrm{PhD}^{2)^{*}}$ \\ 1) Department of Skin and Health Care, Suseong College, Republic of Korea \\ 2) Department of Physical Therapy, Kangwon National University: 3 Hwangjo-ri, Dogye-eup, \\ Samcheok-si, Gangwon-do 245-907, Republic of Korea
}

\begin{abstract}
Purpose] The purpose of this preliminary study was to investigate the effect of manual lymph drainage (MLD) of the neck on frontal electroencephalogram (EEG) asymmetry in subjects with psychological stress. [Subjects] Thirteen subjects with psychological stress participated in the study. [Methods] Subjects received MLD of the neck for $15 \mathrm{~min}$. [Results] Analysis of the frontal asymmetry index showed that the energy shift in the alpha frequency band from the left hemisphere to the right hemisphere after MLD resulted in greater left-side activation (positive asymmetry values), which could be related to the positive emotional state observed particularly in the F7F8 area. [Conclusion] These preliminary findings suggest that frontal EEG asymmetry was significantly attenuated after MLD.

Key words: Stress, Manual lymph drainage, Frontal EEG asymmetry
\end{abstract}

(This article was submitted Sep. 26, 2013, and was accepted Oct. 30, 2013)

\section{INTRODUCTION}

In the field of medicine, "stress" is one of the most common complaints among patients ${ }^{1}$. Stress occurs when an individual fails to respond appropriately to challenge. Stress may negatively influence the affective state of an individual, which in turn may exert direct adverse effects on biological processes or behavioral patterns, thereby increasing the individual's disease risk and pathogenesis of a disease ${ }^{2)}$.

Psychosocial stress is believed to contribute to musculoskeletal disorders of the neck, shoulders, and other areas. Previous studies have shown that mental stress induces a significant increase in muscle tension ${ }^{3)}$. Recently, it has been demonstrated that the same motor units are activated by mental stress as by physical stress, which means that mental stress may also result in low-threshold motor units remaining active during breaks at work and outside of work $^{3)}$. Thus, numerous therapeutic approaches have been used to treat musculoskeletal diseases caused by psychological stress ${ }^{4-6)}$.

The utilization of alternative medicine has been increasing in recent years, with massage documented as being one of the most frequently treatments for musculoskeletal disease $^{7)}$. Manual lymph drainage (MLD) is a procedure that consists of several techniques derived from traditional

*Corresponding author. Sung-Joong Kim (E-mail: lymphkim@kangwon.ac.kr)

(C2014 The Society of Physical Therapy Science. Published by IPEC Inc. This is an open-access article distributed under the terms of the Creative Commons Attribution Non-Commercial No Derivatives (by-ncnd) License $<$ http://creativecommons.org/licenses/by-nc-nd/3.0/>. massage ${ }^{8)}$. MLD has become increasingly popular in recent years, not least because of the enormous amount of publicity it has recieved ${ }^{8-12}$. Moreover, the spread of information about edema among doctors, physical therapists, and patients, and the efforts for managing its cosmetic aspects have also resulted in its popularity ${ }^{10-12)}$.

The frontal region of the brain has been associated with affective states, and different affective states have been associated with different EEG patterns in this region ${ }^{13)}$. Normal subjects experience positively valenced emotions, such as happiness and joy, together with greater relative left frontal EEG activation. In contrast, the experience of negative emotions, including depression, is associated with greater relative right frontal EEG activation ${ }^{14,15)}$. There are some studies that have investigated the effects of different manual techniques on frontal EEG activation $\left.{ }^{4}, 5,16,17\right)$; however, MLD is not one of the techniques that have been investigated. Therefore, the present preliminary study investigated the effects of MLD in subjects with psychological stress and greater relative right frontal EEG activation, with MLD expected to shift EEG patterns toward symmetry.

\section{SUBJECTS AND METHODS}

The subjects included 52 university students, chosen according to the following criteria: (1) no history of mental illness, (2) not currently taking any medication known to affect EEG signals, and (3) no known heart- or musclerelated disease. They were all free of pulmonary, cardiac, and metabolic disease, as well as other disease states, which may cause brain dysfunction. All subjects responded to the stress response questionnaire $(\mathrm{SRI})^{18)}$ and returned it to the 
authors. According to the results of the questionnaire, $13 \mathrm{fe}$ male subjects aged 19 to 23 years with an SRI score $>80$ and a greater relative right frontal EEG activation were included. Informed, written consent was obtained from each subject after the experimental procedures had been explained. All test protocols were approved by the ethics committee of the Physical Therapy Faculty of Kangwon National University.

The participants received a 15-min session of MLD within the neck region. All interventions were completed in a supine position on a massage table with a pillow placed under the knees to relax the lower back muscles. Data acquisition and MLD were performed in a quiet, temperaturecontrolled environment $\left(22-24{ }^{\circ} \mathrm{C}\right)$. Stimuli such as conversation, phone calls, and noise that could increase the activity of sentinel nodes were minimized, and the subject's body was covered with a soft and thin sheet to avoid discomfort from body exposure. MLD was conducted by a well-trained certified MLD therapist, and applied twice to the neck area. Further details of the MLD procedure can be found in $D r$. Vodder's Manual Lymph Drainage: A Practical Guide ${ }^{8)}$. The protocol was standardized, in that, the massage stroke category (type) and time was the same for all participants.

Subjects were allowed to rest comfortably for at least 5 min prior to the baseline recording procedure. EEG data were acquired for $5 \mathrm{~min}$ before, and immediately following, MLD. A total of 6 channels of EEG were recorded: inclusive of Fp1-Fp2, F3-F4, and F7-F8. There were two other electrodes, a ground electrode and reference electrode, which were placed on both zygomatic bones. Participants were then asked to close their eyes and refrain from talking, falling asleep, or making exaggerated body movements, in order to observe the cortical electrical activity without any external stimuli, minimizing possible visual artifacts during EEG measurement. The EEG signal was acquired over a 5-min period, followed by computerized Fourier analysis of the EEG waves using the TeleScan software package (LAXTHA, Daejeon, South Korea). The signal was sampled at a rate of $256 \mathrm{~Hz}$ and was digitally filtered using a $1-50-\mathrm{Hz}$ band-pass filter. After data acquisition and storage, all statistics were computed to extract asymmetry values for the alpha frequency band in the frontal area of the cerebral cortex ${ }^{19-21)}$. The asymmetry index was calculated by subtracting the log-transformed absolute alpha power of the left hemisphere from the analogous log-transformed right hemisphere alpha power (log right - log left). As alpha power is inversely associated with cortical activation, a negative asymmetry score, which denotes greater alpha activity on the left and less alpha power on the right, would suggest greater right-sided activation. On the other hand, a positive score would represent greater activation on the left ${ }^{14,21)}$

All variables were tested for normality using the onesample Kolmogorov-Smirnov test and demonstrated a normal distribution. The asymmetry index was compared prior to, and after, MLD application using a paired t-test, and the collected data were analyzed using a statistical package program (SPSS v. 19.0). A two-tailed probability of $\mathrm{p}<0.05$ was considered statistically significant.
Table 1. Alpha asymmetry scores for each region

\begin{tabular}{lcc}
\hline Area & Before- MLD & After- MLD \\
\hline Fp1-Fp2 & $-0.13 \pm 0.06$ & $0.03 \pm 0.18$ \\
F3-F4 & $-0.15 \pm 0.05$ & $0.02 \pm 0.16$ \\
F7-F8 & $-0.12 \pm 0.04$ & $0.09 \pm 0.25 *$ \\
\hline
\end{tabular}

All variables are means \pm SD, MLD: manual lymph drainage, ${ }^{*} \mathrm{p}<0.05$

\section{RESULTS}

MLD generally caused an activation shift from the right hemisphere to the left hemisphere in all frontal electrode pairs analyzed in this study. Statistical analysis comparing pre- and post-MLD asymmetry values in channels F7-F8 showed a statistically significant increase in hemispheric asymmetry: Fp1-Fp2, p =0.082; F3-F4, p $=0.065$; and F7$\mathrm{F} 8, \mathrm{p}=0.032$. Electrophysiological results from the three frontal electrode pairs are shown in Table 1.

\section{DISCUSSION}

In this preliminary study, frontal lobe EEG activity patterns in response to MLD of the neck were examined to better understand alpha wave processing in subjects with psychological stress. Many studies have shown that EEG asymmetry can be attenuated by massage, relaxation techniques, and musical therapies ${ }^{22-24)}$. However, to date, there are no studies investigating the effects of MLD on frontal asymmetry.

MLD application acts as a very light stimulator against psychological stress; we predicted that MLD would be associated with frontal EEG asymmetry and state, and specifically result in greater left frontal hemisphere activation in the alpha frequency band. In this study, the alpha band (typically in the range of $8-13 \mathrm{~Hz}$ ) was chosen as an electrophysiological marker because, as previously discussed, it is relatively stable ${ }^{21,25)}$ and inversely related to activation $^{26-28)}$. The measurement of alpha asymmetry has been shown to be effective and reliable in discriminating positive and negative emotions ${ }^{23}$,29). Since previous investigations have demonstrated a relationship between brain electrophysiology in the frontal area and measures of affective states $^{19,20,30,31)}$, we have also focused our analysis on the three anterior electrode pairs, Fp1-Fp2, F3-F4, and F7-F8.

Recent studies have suggested that greater left-sided anterior activation, indexed by decreased alpha activity in the left hemisphere, is associated with a higher degree of positive affectivity, a feeling of well-being, and a reduction in anxiety ${ }^{28,32}$. Our results indicate that MLD is associated with a significant increase in left-sided anterior activation, thus supporting our hypothesis that MLD can attenuate frontal EEG asymmetry. These results are also in line with findings from previous studies that have demonstrated the therapeutic effects of massage ${ }^{4,5,20)}$.

Although this study showed demonstrable effects of MLD, there are several limitations and suggestions for future research. Firstly, this study only examined the acute electrophysiological changes produced after MLD in sub- 
jects with psychological stress. In addition to replicating the present findings with a bigger sample size, further studies are needed to investigate the effects of MLD on other clinical populations across different conditions and diseases under a more controlled experimental design.

In conclusion, our preliminary study showed that MLD can significantly increase left-sided anterior activation. These results provide evidence to support the hypothesis that MLD gives rise to positive affectivity. Further investigations will focus on future replication and extension of these preliminary findings.

\section{REFERENCES}

1) Dimsdale JE: Psychological stress and cardiovascular disease. J Am Col Cardiol, 2008, 51: 1237-1246. [Medline] [CrossRef]

2) Cohen S, Kessler RC, Gordon UL: Strategies for measuring stress in studies of psychiatric and physical disorder. In: Cohen S, Kessler RC, Gordon UL, eds. Measuring Stress: A Guide for Health and Social Scientists. New York: Oxford University Press, 1955, pp 3-26.

3) Lundberg U: [Psychological stress and musculoskeletal disorders: psychobiological mechanisms. Lack of rest and recovery greater problem than workload]. Lakartidningen, 2003, 100: 1892-1895. [Medline]

4) Field T, Grizzle N, Scafidi F, et al.: Massage and relaxation therapies' effects on depressed adolescent mothers. Adolescence, 1996, 31: 903-911. [Medline]

5) Jones NA, Field T, Davalos M: Massage therapy attenuates right frontal EEG asymmetry in one-month-old infants of depressed mothers. Infant Behav Dev, 1998, 21: 527-530. [CrossRef]

6) Stanley MA, Diefenbach GJ, Hopko DR: Cognitive behavioral treatment for older adults with generalized anxiety disorder. A therapist manual for primary care settings. Behav Modif, 2004, 28: 73-117. [Medline] [CrossRef]

7) Buttagat V, Eungpinichpong W, Kaber D, et al.: Acute effects of traditional Thai massage on electroencephalogram in patients with scapulocostal syndrome. Complement Ther Med, 2012, 20: 167-174. [Medline] [CrossRef]

8) Wittlinger H, Wittlinger D, Wittlinger A, et al.: Dr. Vodder's Manua Lymph Drainage - A Practical Guide- . New York: Thieme, 2011, pp 36-38.

9) Chikly B: Theory of Practice of Lymph Drainage Therapy. Arizona: I. H. H. Publishing, 2001, pp 231-284.

10) Kasseroller RG: The Vodder School: the Vodder method. Cancer, 1998, 83 2840-2842. [Medline] [CrossRef]

11) Weissleder H, Schuchhardt C: Lymphedeam: Diagnosis and Therapy. Koln: Viavital Verlag GmbH, 2008, pp 403-423.

12) Cavezzi A, Michelini S: Phlebolymphoedema - From diagnosis to therapy-. Bologna: Edizioni P.R., 1998, pp 55-65.

13) Fox NA: If it's not left, it's right. Electroencephalograph asymmetry and the development of emotion. Am Psychol, 1991, 46: 863-872. [Medline] [CrossRef]

14) Davidson RJ: EEG measures of cerebral asymmetry: conceptual and meth- odological issues. Int J Neurosci, 1988, 39: 71-89. [Medline] [CrossRef]

15) Schmidt LA, Trainor LJ, Santesso DL: Development of frontal electroencephalogram (EEG) and heart rate (ECG) responses to affective musical stimuli during the first 12 months of post-natal life. Brain Cogn, 2003, 52: 27-32. [Medline] [CrossRef]

16) Jones NA, Field T: Massage and music therapies attenuate frontal EEG asymmetry in depressed adolescents. Adolescence, 1999, 34: 529-534. [Medline]

17) Tornek A, Field T, Hernandez-Reif M, et al.: Music effects on EEG in intrusive and withdrawn mothers with depressive symptoms. Psychiatry, 2003, 66: 234-243. [Medline]

18) Koh KB, Park JK, Kim CH: Development of the Stress Response Inventory. J Korean Neuropsychiatr Assoc, 2000, 39: 707-719.

19) Harmon-Jones E, Allen JJ: Behavioral activation sensitivity and resting frontal EEG asymmetry: covariation of putative indicators related to risk for mood disorders. J Abnorm Psychol, 1997, 106: 159-163. [Medline] [CrossRef]

20) Sutton SK, Davidson RJ: Prefrontal brain electrical asymmetry predicts the evaluation of affective stimuli. Neuropsychologia, 2000, 38: 17231733. [Medline] [CrossRef]

21) Tomarken AJ, Davidson RJ, Wheeler RE, et al.: Psychometric properties of resting anterior EEG asymmetry: temporal stability and internal consistency. Psychophysiology, 1992, 29: 576-592. [Medline] [CrossRef]

22) Field $T$, Ironson $G$, Scafidi $F$, et al.: Massage therapy reduces anxiety and enhances EEG pattern of alertness and math computations. Int J Neurosci, 1996, 86: 197-205. [Medline] [CrossRef]

23) Field T, Martinez A, Nawrocki T, et al.: Music shifts frontal EEG in depressed adolescents. Adolescence, 1998, 33: 109-116. [Medline]

24) Chan AS, Han YM, Cheung MC: Electroencephalographic (EEG) measurements of mindfulness-based Triarchic body-pathway relaxation technique: a pilot study. Appl Psychophysiol Biofeedback, 2008, 33: 39-47. [Medline] [CrossRef]

25) Davidson RJ, Ekman P, Saron CD, et al.: Approach-withdrawal and cerebral asymmetry: emotional expression and brain physiology. I. J Pers Soc Psychol, 1990, 58: 330-341. [Medline] [CrossRef]

26) Klimesch W: EEG alpha and theta oscillations reflect cognitive and memory performance: a review and analysis. Brain Res Brain Res Rev, 1999, 29: 169-195. [Medline] [CrossRef]

27) Pivik RT, Broughton RJ, Coppola R, et al.: Guidelines for the recording and quantitative analysis of electroencephalographic activity in research contexts. Psychophysiology, 1993, 30: 547-558. [Medline] [CrossRef]

28) Urry HL, Nitschke JB, Dolski I, et al.: Making a life worth living: neural correlates of well-being. Psychol Sci, 2004, 15: 367-372. [Medline] [CrossRef]

29) Davidson RJ, Kabat-Zinn J, Schumacher J, et al.: Alterations in brain and immune function produced by mindfulness meditation. Psychosom Med, 2003, 65: 564-570. [Medline] [CrossRef]

30) Davidson RJ: Anterior cerebral asymmetry and the nature of emotion. Brain Cogn, 1992, 20: 125-151. [Medline] [CrossRef]

31) Davidson RJ: Asymmetric brain function, affective style and psychopathology: the role of early experience and plasticity. Dev Psychopathol, 1994, 6: 741-758. [CrossRef]

32) Davidson RJ: Affective style, psychopathology, and resilience: brain mechanisms and plasticity. Am Psychol, 2000, 55: 1196-1214. [Medline] [CrossRef] 